\title{
Computational fluid dynamics evaluation of conditions before impact of particles in plasma spraying process
}

\author{
KJ Mbwebwe ${ }^{1 a}$, A Kolesnikov ${ }^{1 b}$, IJ Van Der Walt ${ }^{2 c}$, H Bissett ${ }^{2 d}$ \\ ${ }^{1}$ Department of Chemical, Metallurgical and Materials Engineering, Tshwane University of Technology, Pretoria 0185, South Africa \\ ${ }^{2}$ The South African Nuclear Energy Corporation SOC Ltd. (Necsa), Elias Motsoaledi Street Extension (Church Street West) R104 Pelindaba, \\ Madibeng Municipality, North West Province, 0240, South Africa

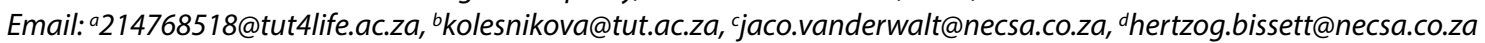

\begin{abstract}
Plasma Spraying is one of the most sophisticated and versatile thermal spray techniques. In Plasma Spraying, powdered material is injected into a plasma jet, which is generated from a plasma torch. Upon contact with the plasma jet, the particles are melted and propelled forward onto a substrate to form an adherent coating which modifies the properties of the substrate. The modifications to the substrate can, for example, increase its resistance to other extreme operating conditions such as wear, abrasion, and corrosion. However, the phenomena governing the formation of the plasma jet inside the plasma torch and its subsequent interaction with injected particles are not fully understood. This paper provides a detailed report on steps taken for the development of a comprehensive numerical model to simulate plasma jet development inside a direct current plasma torch. The heat flow and mass exchange of ionized gas with injected solid particles were followed in three dimensions by using a Computational Fluid Dynamics (CFD) method. A cylindrical energy source term which was defined as an increasing linear function dependent on time as a variable, was included to reproduce the effects of an electric arc on the gas flow. For optimization purposes, it was sought to investigate the effects of the particles' injection angle and inlet velocity, as well as the effects of particle size distribution on the particle temperature and velocity histories.
\end{abstract}

Keywords: Plasma jet, Plasma spraying, Temperature, Velocity, Particles

\section{Introduction}

The coating of surfaces by plasma spraying is an important manufacturing process with many industrial applications (Vardelle et al, 2001), including wear, heat and corrosion resistance, surface restoration, and others required in aircraft, automobile, power plant, oil, and gas industries (Sahab et al, 2012). In Plasma spraying, feeding stocks (solid particles) are injected into a high temperature, high-velocity thermal plasma jet, in which they are heated, accelerated, and deposited to form coatings (Zhang et al, 2007).

The process parameters required to produce sound and reproducible coatings are usually associated with the spray equipment (Vardelle et al, 2001), and several other variables including the powder feedstock, material injection, and processing variables (Sahab et al, 2012). Most particularly, acceleration and heating of particles are crucial in thermal spraying, to both process efficiency and coating quality, as particles need to achieve a specific range of thermal and kinetic energy (Khelfi et al, 2008).

Due to the complexity of the above-mentioned variables, numerical modelling of the plasma spray process is required for optimizing spray parameters and improving our understanding of complex heat, momentum, and mass transport phenomena involved (Jayasingh et al, 2014). This paper focuses on developing a comprehensive numerical model to simulate plasma jet formation inside a direct current (DC) plasma torch and following heat flow and mass exchange between injected particles and ionized gas. Furthermore, an optimization study is undertaken, and it encompasses (1) an

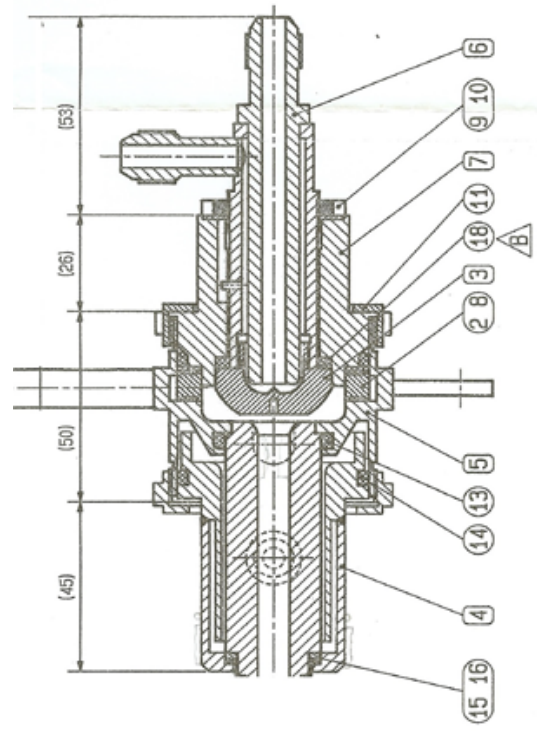

Figure 1: Plasma torch design

investigation of temperature and velocity distributions of the plasma jet; (2) the effect of particle injection angle; (3) the effect of the plasma gas inlet flow rate; (4) a study on the effect of particle size distribution; and (5) the effect of particle carrier gas flow rate or inlet velocity.

\section{Methodology}

\subsection{Physical Model}

Taking the plasma torch in Figure 1 as basis, the physical model for the envisaged study was configured. It follows that the copper 
Table 1: Conservation Equations

\begin{tabular}{llll} 
& $\varnothing$ & $\Gamma \emptyset$ & $\mathrm{S} \emptyset$ \\
\hline Mass (1) & 1 & 0 & 0 \\
Momentum (2) & $\vec{V}$ & $\mu$ & $\vec{J} \cdot \vec{B}$ \\
Energy (3) & $\mathrm{h}$ & $\frac{k}{C p}$ & $\frac{5}{2} \frac{\mathrm{Kb}}{e}\left(\frac{\vec{J}}{C p} \cdot \nabla h\right)+\vec{J} \cdot \vec{E}-S r$ \\
\hline
\end{tabular}

cathode is half spherical with a circular base and top of respective diameters, $14.5 \mathrm{~mm}$, and $8 \mathrm{~mm}$. As for the copper nozzle (anode), it has a diameter of $4 \mathrm{~mm}$ and is $91 \mathrm{~mm}$ long. To produce a plasma discharge, the torch was supplied with a DC power of up to $30 \mathrm{~kW}$ and with a thermal efficiency of $60 \%$. Finally, the total gas flow through the torch was about $3 \mathrm{~kg} / \mathrm{h}$.

\subsection{Plasma Jet Model}

The plasma jet was simulated by assuming that it was steady, in local thermodynamic equilibrium (LTE), optically thin, incompressible, turbulent, and that its mass diffusivity was equal to the thermal diffusivity (Selvan and Ramachandran, 2009). In addition, the transport and thermodynamic properties of argon plasma were functions of the local temperature and were calculated based on the data reported in Boulos et al (1994).

Based on the aforementioned assumptions, the conservation equations of mass, momentum, and energy, were generated, as listed in Table 1, which shows the dependent property ( $\varnothing$ ), diffusion coefficient $\left(\Gamma_{ø}\right)$, and source term $\left(\mathrm{S}_{\wp}\right)$ for each equation. In Table 1 , $\vec{V}, \mathrm{~h}, \mu, \mathrm{k}, \vec{J}, \mathrm{Cp}, \mathrm{Kb}, \mathrm{e}$, and $\mathrm{Sr}$ are, respectively, velocity vector, enthalpy, viscosity, thermal conductivity, current density vector, specific heat, Boltzman's constant, elementary electric charge, and volumetric radiation power (Kim et al, 2008). The determined governing equations are solved by the SIMPLE algorithm used in commercial CFD software ANSYS FLUENT R21.

\subsection{Computational Domain and Boundary Conditions}

A fully structured three-dimensional mesh of 1.48 million cells was employed to model the fluid domain of the non-transferred DC plasma torch and outside domain as shown in Figure 2. Argon gas was selected as the plasma fluid and was injected into the torch through 4 tangential inlets of diameter $2 \mathrm{~mm}$. These tangential inlets form a swirling effect throughout the anode nozzle, as it provides stabilization to the generated plasma.

Non-slip boundary conditions were forced on all solid walls. The temperature on these walls is presumed to be $300 \mathrm{~K}$ except on the cathode and the anode. The inner side of the anode being close enough to the high-temperature plasma jet and its outer side being cooled by water, a heat transfer coefficient of $10^{5} \mathrm{~W} / \mathrm{m}^{2}-\mathrm{K}$ was imposed on the cathode. On the other hand, the temperature of the cathode is assumed to $\mathrm{T}=3500 \mathrm{~K}$. Zero electric potential and current density are imposed on all solid boundaries, including the cathode, as a plasma source term is included in the anode nozzle to simulate electric arc effects on the plasma jet. Finally, the temperature at the inlet was $300 \mathrm{~K}$ and the argon gas inlet flow rate was fixed at $3 \mathrm{~kg} / \mathrm{h}$.

\subsection{Discrete Phase Modelling}

The Discrete Phase Model (DPM) provided in ANSYS FLUENT $\mathrm{R} 21$, is a model specially developed for spray simulations, or more generally for suspended particle trajectory simulations. It is based on the Euler-Lagrange method whereby the computational domain has two separate phases, the continuous and the discrete phase (particles). The fluid phase is treated as a continuum by solving the Navier-Stokes equations, while the dispersed phase is solved by tracking a large number of particles through the calculated flow field (Jayasingh et al, 2014).

Momentum and energy transfer are the basic interaction mechanisms between powder particles and plasma jet. Through momentum interactions, it is possible to determine the velocity and trajectory of powder particles. The analysis of the energy transfer

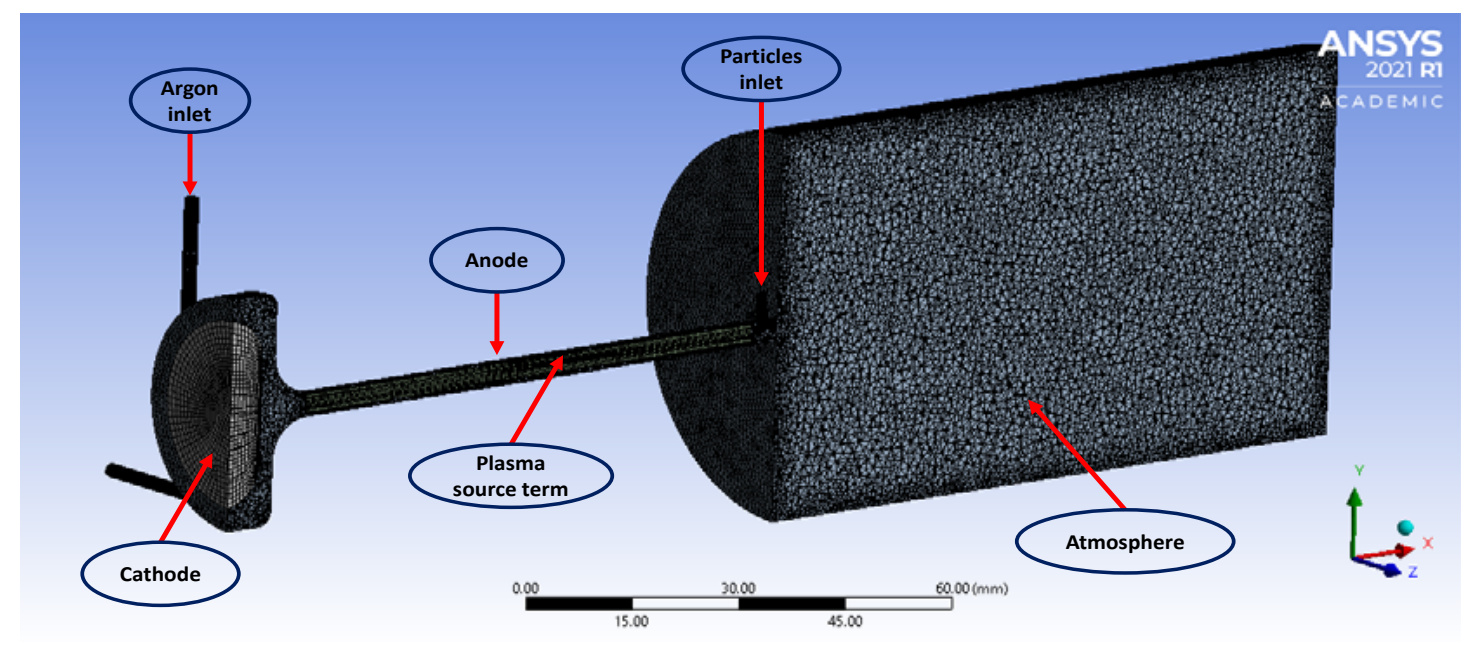

Figure 2: Mesh of computational domain cross-section 

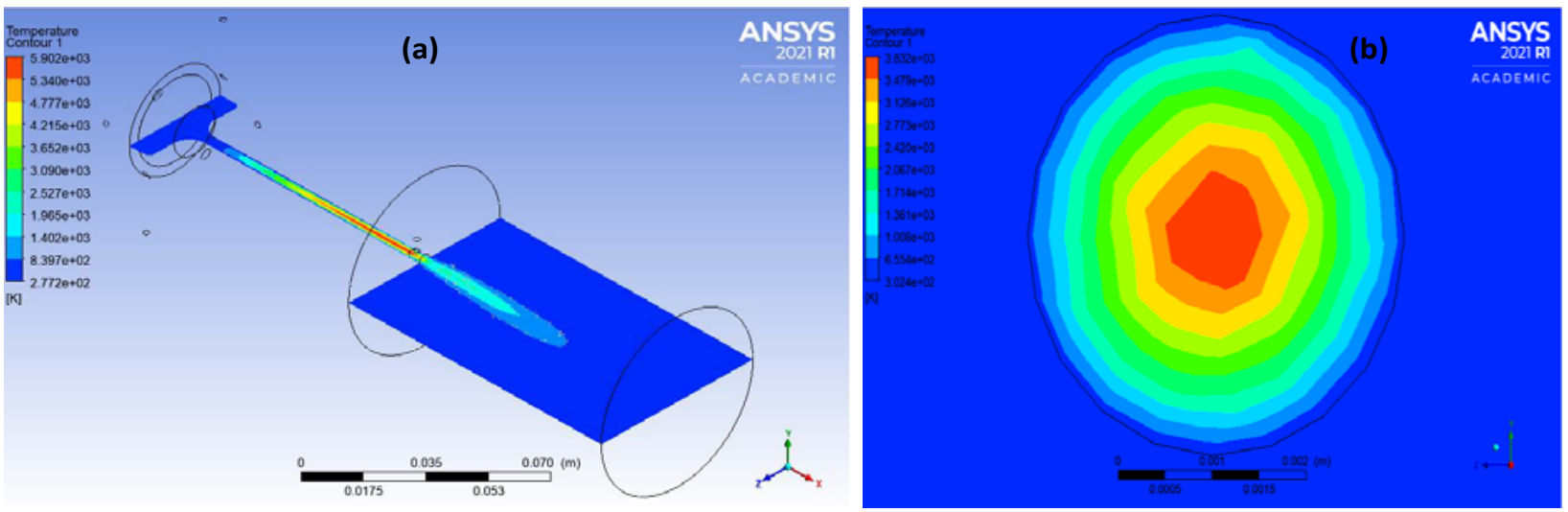

Figure 3: Temperature distribution of plasma jet in (a) isomeric direction (b) radial direction at the torch exit

between the powder particles and plasma jet can be applied for the description of temperature and phase changes of powder particles (Kotowski et al, 2012).

To generate particle trajectory and thermal history equations and simulation results, the use of appropriate assumptions is necessary. In this study, titanium powder was injected internally (downstream the plasma torch) by an argon carrier gas in the plasma jet. Particles in a powder, having different diameters, were assumed to follow the Rosin-Rammler diameter distribution method. To further simplify the model, the particles were assumed to be spherical and to have a velocity derived from that of the carrier gas in the plasma jet. In addition, particles' internal temperature was uniform, and they were not interacting with each other. Furthermore, the carrier gas flow rate did not vary with time, the injector walls were smooth and straight, and the velocity of the carrier gas was not time-dependent and was constant (Delluc et al, 2005; Jayasingh et al, 2014).

\section{Results and Discussion}

\subsection{Temperature and Velocity distributions of plasma jet}

Figure 3 and Figure 4 show the temperature and velocity distributions of the plasma jet over the whole computational domain and at the torch outlet for an initial primary argon flow rate of $3 \mathrm{~kg} / \mathrm{h}$ under atmospheric pressure and subjected to a power of $30 \mathrm{~kW}$.

Figure 3 (a) shows that, as expected, heat generation is maximum in the plasma source term area downstream the anode nozzle, generating temperatures of the order of $5902 \mathrm{~K}$. However, it can be observed that as the plasma jet discharges into the open atmosphere, the temperature gradually decreases as it gets further away from the plasma source. From the particles heating and melting perspective, two thermal characteristics of the plasma jet are of interest: firstly, as the particle injection is internal and $3 \mathrm{~mm}$ from the torch exit, the radial temperature distribution at the torch exit is of utmost importance. Secondly, the thermal history of the plasma jet from the time it exits the torch to before impact on the substrate. Investigation of Figure 3 (b) reveals strong temperature gradients from the plasma core to the plume extremities, ranging from $3832 \mathrm{~K}$ to $654 \mathrm{~K}$. Nevertheless, since the plasma core of temperature $3832 \mathrm{~K}$ occupies quite a considerable area compared to other temperature layers at the exit, its temperature should be enough to heat and melt titanium particles, whose melting temperature is 1941 K. On the other hand, a closer look at Figure 3 (a) shows that temperature rapidly decreases (to $840 \mathrm{~K}$ ) as the distance from to torch exit increases, due to heat conduction and convection with the atmosphere. This could be a challenge as it would negatively affect the effective heating and melting of particles. Hence, parameter variation will be undertaken in the upcoming sections to ensure variables are optimized for better performance.

Figure 4 shows that velocity reaches a maximum value of 1758 $\mathrm{m} / \mathrm{s}$ which is in accord with studies on Numerical (CFD) Analysis of Thermal Spray Coating Process conducted by Jayasingh et al. (2014), whereby the gas-phase maximum velocity was $1800 \mathrm{~m} / \mathrm{s}$. Figure 4 (a) shows that the maximum velocity is reached close to the torch outlet but outside the torch, while Figure 4 (b) shows
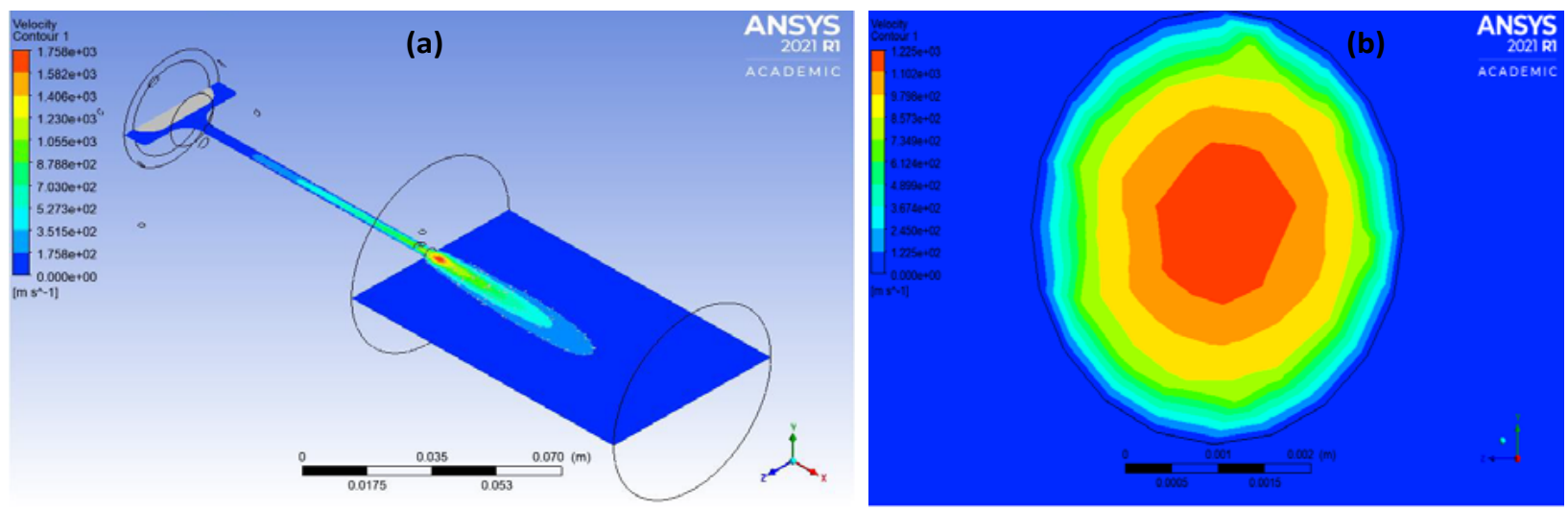

Figure 4: Velocity distribution of plasma jet in (a) isomeric direction (b) radial direction at the torch exit 


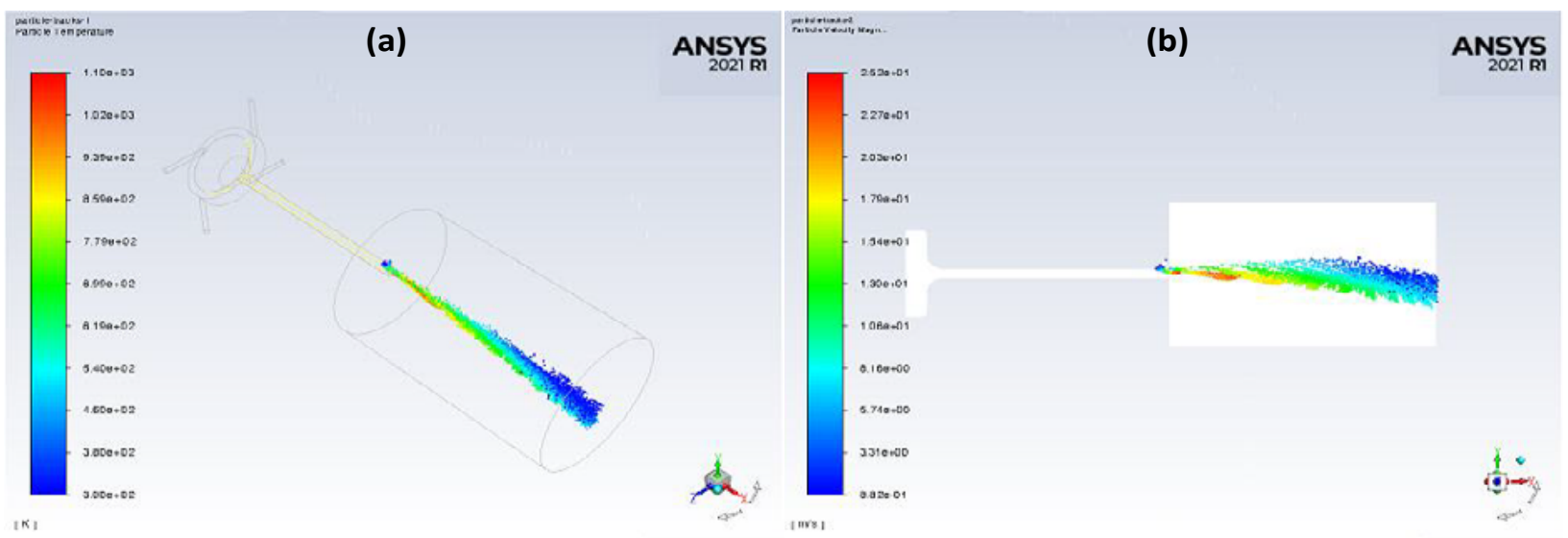

Figure 5: Particle-tracking at (Time $=0.25 \mathrm{~s})$ of (a) particle temperature in isomeric direction (b) particle velocity in axial direction

that the outlet velocity profile is more uniform than the temperature profile. This is owed to the fact that the velocity being dependent on density, the change in velocity profile is slower and smoother compared to the temperature (Ardakani, 2016).

\subsection{Particle Tracking and Effect of Particle Injection Angle}

The titanium particle inlet velocity was $3.025 \mathrm{~m} / \mathrm{s}$ and the particle diameter range, mean particle diameter, and the carrier gas mass flow rate were $20-40 \mu \mathrm{m}, 30 \mu \mathrm{m}$, and $0.75 \mathrm{~kg} / \mathrm{h}$, respectively.

Figure 5 (a) and (b) show particle tracking during simulation of particle temperature and particle velocity, at a simulation time equivalent to $0.25 \mathrm{~s}$.

For optimization of the particle injection angle, the following three types of injection angles were used: the vertical position, the $45^{\circ}$ upstream inclined injection and the $45^{\circ}$ downstream inclined injection. The particle injection velocity is kept constant at $3.025 \mathrm{~m} / \mathrm{s}$ for all cases. Investigation of Figure 6 (b) shows that the upstream inclined injection increases the particle velocity as it moves towards the substrate, although it acquires a lower initial axial velocity due to particles being injected in a counter flow configuration. The downstream injection yields the lowest particle velocity profile because it has the shortest contact time. On the other hand, Figure 6 (a) shows that the upstream injection generates higher temperature values compared to others. This verifies the modelling work carried out by Khefi et al. (2008), who found similar trends.

(a)

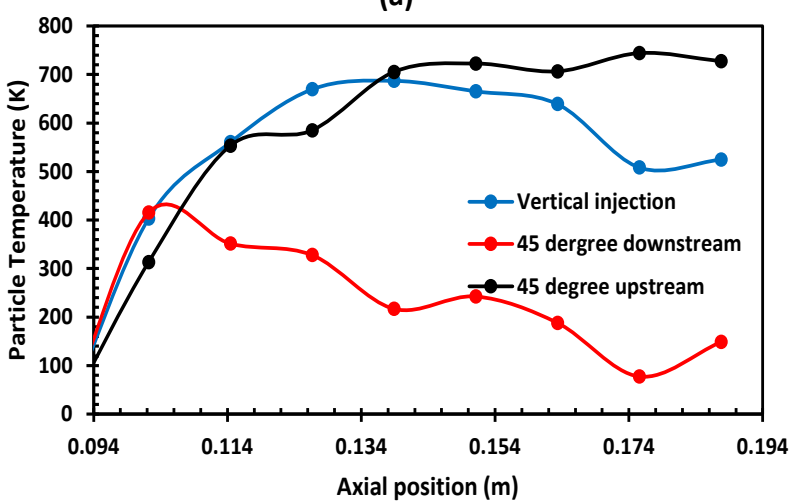

Figure 6: Effect of particle injection angle on (a) particle temperature (b) particle velocity

\subsection{Effect of Particle Size Distribution}

Figure 7 shows temperature and velocity distributions for three different particle sizes: $30 \mu \mathrm{m}, 40 \mu \mathrm{m}$, and $50 \mu \mathrm{m}$, respectively. The first observation made is that particles of different sizes have different velocities and temperatures. Secondly, it is observed that the velocity of particles decreases with an increase in particle size. This is due to particle acceleration dependency on particle weight. Implying that lighter particles reach higher velocity as their opposition to the plasma jet movement is negligible compared to heavier particles as attested by the works conducted by Delluc et al. (2005) and Jayasingh et al. (2014). Correspondingly, lighter particles yield higher temperatures.

\subsection{Effect of Primary Gas Inlet Flow Rate}

Three sets of operating conditions with different inlet gas flow rates $(2,3$, and $4 \mathrm{~kg} / \mathrm{h})$ have been simulated to investigate the influence of inlet gas flow rate on the particle temperature and velocity. Figure 8 (a) reveals that a decrease in argon inlet gas flow rate induces higher particle temperatures and velocities. This is due to the fact that as the argon gas flow rate is decreased, there is greater energy allocation per plasma species, favouring higher energetic levels, thus higher temperatures. Figure 8 (b) shows that as the inlet argon gas is decreased, particle velocity is decreased as well seen as there is lower momentum transferred from the plasma gas to in-flight particles as also concluded by Zhang et al. (2007) through his modelling and experimental work on the effects of injection angle and carrier gas flow rate on in-flight characteristics in Plasma

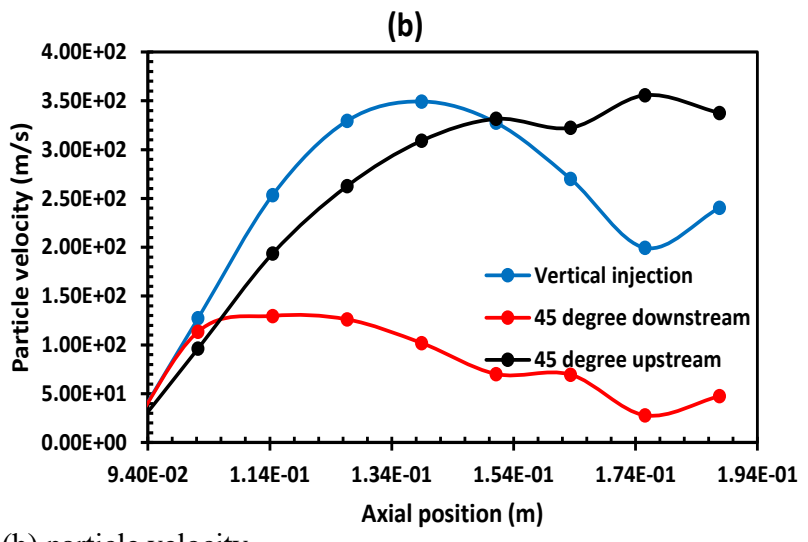


(a)

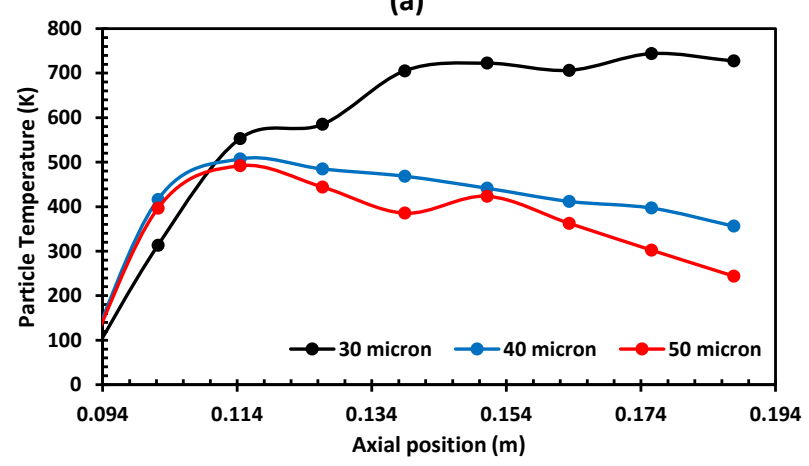

(b)

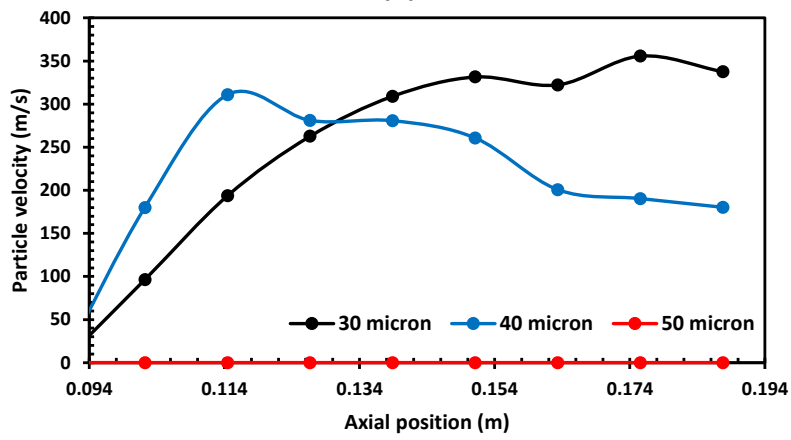

Figure 7: Effect of particle size distribution on (a) particle temperature (b) particle velocity

(a)

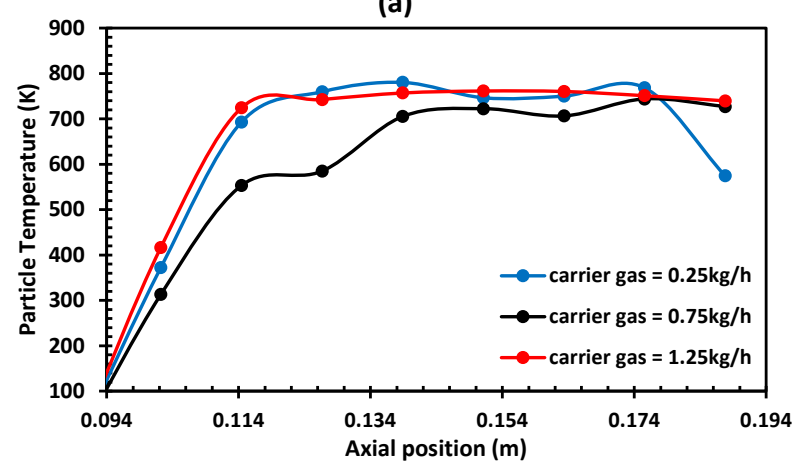

(b)

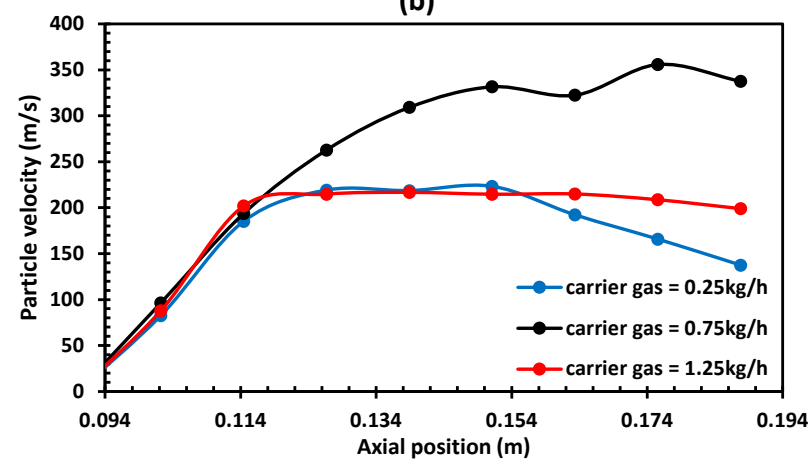

Figure 8: Effect of plasma gas inlet flowrate on (a) particle temperature (b) particle velocity

Spray Process. However, the trend for particle temperature at an inlet argon flow rate of $4 \mathrm{~kg} / \mathrm{h}$ defies the above observation as opposed to the two other flow rates. Additional simulation work should be carried out to confirm this behaviour, to further discuss its probable causes.

\subsection{Effect of Carrier Gas Flowrate (Inlet Velocity)}

To investigate the influence of carrier gas flow rate variation on particle temperature and velocity, simulations have been performed with 3 carrier gas flow rates $(0.25,0.75$, and $1.25 \mathrm{~kg} / \mathrm{h})$ while maintaining all the other variables constant, specifically the inlet argon gas flow rate at its initial value of $3 \mathrm{~kg} / \mathrm{h}$. The velocity of particles having been assumed to derive from the carrier gas flow rate, the carrier gas flow rate is expected to have a behaviour strongly correlated to that of the powder feed rate. Figure 9 (b) shows that the carrier gas flow rate of $0.25 \mathrm{~kg} / \mathrm{h}$ generates the lowest final velocity $(137 \mathrm{~m} / \mathrm{s})$ compared to the two other flow rates, indicating that the flow rate is too low, therefore hindering particles penetration into the plasma jet. Subsequently, its corresponding temperature is the lowest as shown in Figure 9 (a). It appears that the medium carrier gas flow rate, $0.75 \mathrm{~kg} / \mathrm{h}$, generates the highest final velocity $(337 \mathrm{~m} / \mathrm{s})$ and has an identical final temperature as the one generated by the carrier gas flow rate of $1.25 \mathrm{~kg} / \mathrm{h}$. However, the latter, despite having a lower final velocity $(198.9 \mathrm{~m} / \mathrm{s})$, appears to have the finest profile in terms of steadiness, for both particle temperature and velocity. Hence, the optimum carrier gas flow rate for the current conditions lies between $0.75-1.25 \mathrm{~kg} / \mathrm{h}$. Its exact value should be determined by more extensive simulation runs in the determined range. The carrier gas mass flow rate must be adjusted so that the maximum number of particles penetrate the plasma flow and acquire optimum acceleration and heating. (a)

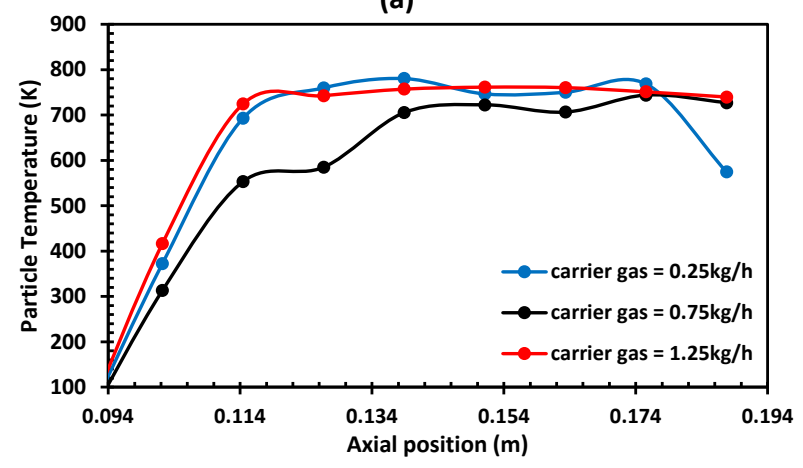

(b)

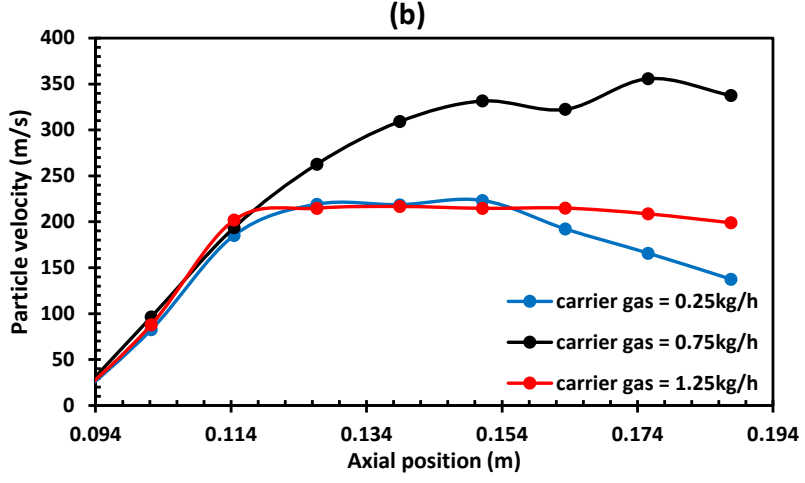

Figure 9: Effect of carrier gas flow rate on (a) particle temperature (b) particle velocity 


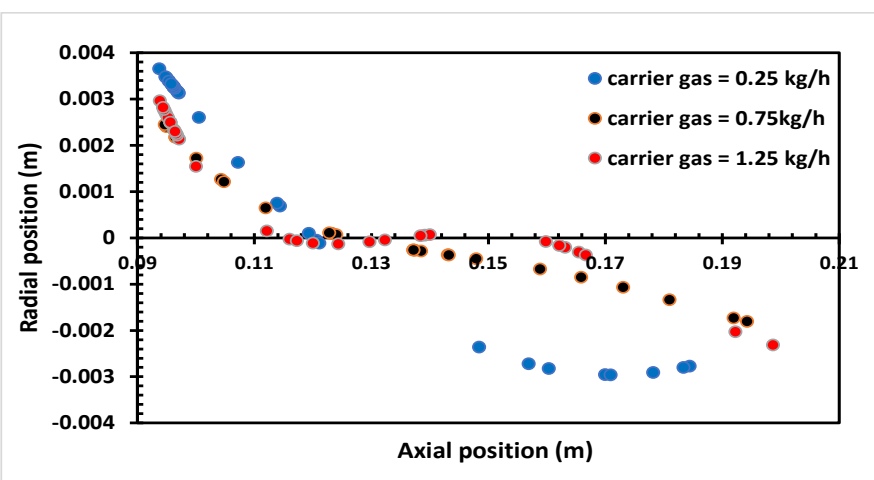

Figure 10: Particle radial distribution at various carrier gas flow rates

As for particle trajectory through the tail flame, Figure 10 shows particle trajectories for carrier gas flow rates of $0.25 \mathrm{~kg} / \mathrm{h}, 0.75 \mathrm{~kg} / \mathrm{h}$ and $1.25 \mathrm{~kg} / \mathrm{h}$, respectively. At $0.25 \mathrm{~kg} / \mathrm{h}$, particles are expected to have a low velocity, preventing effective particle penetration. However, observation of Figure 10 reveals that particles at $0.25 \mathrm{~kg} / \mathrm{h}$ seem to pass through the plasma tail flame at a higher intensity than the two other carrier gas flow rates. This is in contradiction with expected behaviour. The apparent contradiction could result from the fact that particles not penetrating the plasma tail flame build up at the injector, overflowing and free falling to the ground due to gravity. Hence, this gives the impression that particles pass through the tail flame.

On the other hand, comparing particle radial distribution at carrier gas flow rate of $0.75 \mathrm{~kg} / \mathrm{h}$ and $1.25 \mathrm{~kg} / \mathrm{h}$ reveals that the later, having a higher initial velocity passes through the tail flame faster.

\section{Conclusion}

A three-dimensional plasma torch model was developed to simulate plasma jet formation and discharge as well as plasma-particles interactions. Temperature and velocity distributions of the plasma jet were generated, reaching values up to $5902 \mathrm{~K}$ and $1758 \mathrm{~m} / \mathrm{s}$, respectively. To optimize parameters, a comparison between threeparticle injection configurations (vertical, $45^{\circ}$ upstream, and $45^{\circ}$ downstream) was conducted. It resulted in the upstream injection method being more ideal as it led to higher particle temperature and velocity. On the other hand, a study of the effect of particle size distribution on the particle temperature and velocity concluded that an increase in particle size led to a decrease in particle temperature and velocity. In addition, an investigation of the effect of inlet argon gas flow rate on particle temperature and velocity showed that lower inlet argon flow rates contributed to higher particle temperature and velocity, as it allowed better penetration of particles into the plasma jet. Furthermore, it was determined that the optimum carrier gas flow rate, under the operating conditions of this study lied between $0.75-1.25 \mathrm{~kg} / \mathrm{h}$.

\section{Acknowledgements}

The authors would like to acknowledge the Nuclear Materials Development Network (NMDN) of the Advanced Materials Initiative (AMI), funded by the Department of Science and Innovation (DSI) for the financial support in conducting this study. The South African Nuclear Energy Corporation SOC Ltd. (Necsa) is acknowledged for their financial support.

\section{References}

1. Ardakani, E.S., 2016. Numerical and Experimental Study of the Arc Fluctuations in a DC Plasma Torch. Ph.D. Thesis.

2. Boulos, M.I., Fauchais, P., Pfender, E., 1994. Thermal Plasmas: Fundamentals and Applications, Volume 1, New York.

3. Delluc, G., Ageorges, H., Pateyron, B., Fauchais, P., 2005. Modeling of plasma jet and particle behavior in spraying conditions. High-Temperature Material Processes, 1-25.

4. Han, H., Wang, Q., 2012. Three-dimensional arc modeling inside the non-transferred plasma torch. Advanced Materials and Process Technology 217, 1407-1410.

5. Jayasingh, T.R., Jeyaseelan, T.R., Kannan, C., Karthikeyan, M.G., 2014. Numerical (CFD) Analysis of Thermal Spray Coating Process. International Journal of Modern Engineering Research (IJMER) 4(3), 46-62.

6. KhefI, D., Abdellah El-Hadj, A., Ait-Messoundene, N., 2008 Modeling of a 3D plasma thermal spraying and the effect of the particle injection angle. Revue des Energies Renouvelables CISM'08 Oum El Bouaghi, 205-216.

7. Kim, K.S., Park, J.M., Choi, S., Kim, J., Hong, S.H., 2008 Comparative study of two- and three-dimensional modeling arc discharge phenomena inside a thermal plasma torch with hollow electrodes. Physics of Plasmas 15, 1-13

8. Kotowski, S., Sieniawski, J., Goral, M., 2012. Modeling and simulation of plasma spraying process with a use of Jets\&Poudres program. Journal of Achievements in Materials and Manufacturing Engineering 55(2), 547-550.

9. Sahab, A.R.M., Saad, N.H., Kasolang, S., Saedon, J., 2012. Impact of Plasma Spray Variables Parameters on Mechanical and Wear Behaviour of Plasma Sprayed A12O3\%wt TiO2 Coating in Abrasion and Erosion Application. Procedia Engineering 41, 1689-1695.

10. Selvan, B., Ramachandran, K., 2009. Comparisons Between Two Different Three-Dimensional Arc Plasma Torch Simulations. Journal of Thermal Spray Technology 18(5-6), 846-857.

11. Vardelle, M., Vardelle, A., Fauchais, P., Li, K.1., Themelis, N.J., 2001. Controlling Particle Injection in Plasma Spraying. Journal of Thermal Spray Technology 10, 267-284.

12. Zhang, W., Zheng, L.L., Zhang, H., Sampath, S., 2007. Study of Injection Angle and Carrier Gas Flow Rate on Particles In-Flight Characteristics in Plasma Spray Process: Modeling and Experiments. Plasma Chem Plasma Process 27, 701-716. 